\title{
Knowledge and Practices of Hand Washing and Glove Utilization Among the Health Care Providers of Shenen Gibe Hospital, South West Ethiopia
}

\author{
Birhanu Stotie Alemu', Asmamaw Demis Bezune', Jophin Joseph", Addis Adera Gebru ${ }^{1}$, , \\ Yonas Yimam Ayene ${ }^{2}$, Birhan Alemnew Tamene ${ }^{2}$ \\ ${ }^{1}$ Department of Nursing and Midwifery, College of Public Health and Medical Science, Jimma University, Jimma, Ethiopia \\ ${ }^{2}$ Department of Nursing, Faculty of Health Sciences, Woldia University, North Wollo, Amhara Region, Ethiopia
}

\section{Email address:}

sebletefera@gmail.com (S. A. Birhanu), asmamawdemis@gmail.com (D. B. Asmamaw), addisaderagebru@gmail.com (A. A. Gebru), Yonasyimam@gmail.com (Y. A. Yonnas), birhanalemnew12@gmail.com (A. T. Birhan)

\section{To cite this article:}

Birhanu Stotie Alemu, Asmamaw Demis Bezune, Jophin Joseph, Addis Adera Gebru, YonasYimam Ayene, Birhan Alemnew Tamene. Knowldge and Practices of Hand-Washing and Glove Utilization Among the Health Care Providers of Shenen Gibe Hospital, South West Ethiopia. Science Journal of Public Health. Vol. 3, No. 3, 2015, pp. 391-397. doi: 10.11648/j.sjph.20150303.24

\begin{abstract}
Background Nosocomial or Hospital Acquired infections-Is an infection that is neither present nor incubating at the time the patient came to the health care facility(hospital). Nosocomial refers to the association between care and the subsequent onset of infection. To prevents this infection open hand washing and glove utilization practice is simple and an important method. Objectives: To investigate the knowledge and practice of hand washing and glove utilization among the health care workers of Shenen Gibe Hospital. Method: Acrosssectional study design was used to investigate the knowledge and practice of hand washing and glove utilization among the health care provider of Shenen Gibe Hospital. A structured selfadministered pre-tested questionnaire was used for all 47 health professionals by Universal sampling techniques. Results: All the 47 staffs have responded to the questionnaire and the distribution of the staff was: $7(14.8 \%)$ physicians, 3(6.4\%) were BSc Nurses, 21(44.8\%) was clinical nurse, $8(17 \%)$ were mid wifery nurse, $5(10.6 \%)$ labtech. and the rest were anesthetist $3(6.4 \%)$. From this $15(31.9 \%)$ were males and 32(68.1\%) were females. From 47 respondents, 39(82.97\%) and 32(68.08\%) of them have adequate knowledge and practice of hand washing and glove utilization respectively. From the total of 47 respondents, $24(51 \%)$ of them have complied with the recommended procedures of hand washing and glove utilization, while $23(49 \%)$ of them did not comply. Conclusion and Recommendation: Majority of the study subjects have adequate knowledge and practice about hand washing and glove utilization. Most of the respondents were compiled with the recommended procedures of hand washing and glove utilization. Regular practice of hand washing and glove utilization requires regular supplies like soap, water, dry \&clean to well and acceptable glove depending on the type of procedure to perform at all time. Therefore oromia health bureau should facilitate these facilities in the hospital wards. The hospital authorities are urged to post the general guidelines for the staff a teach hand washing sites.
\end{abstract}

Keywords: Nosocomial Infections, Hand Washing, Glove Utilization, Shenen Gibe Hospital

\section{Back Ground}

Nosocomial or hospital acquired infections is infection that is neither present nor incubating at the time the patient came to the health care facility (Hospital). Nosocomial refers to the association between care and the subsequent onset of infection and nosocomial infections are a significant problem throughout the world and are increasing $(1,2)$. Ever since workers who are identified the problem of infection acquired in Hospital, at tempts have been made to eradicate or at least reduce rates of acquisition. Hospital acquired infection, also known as nosocomial infection, is defined as an infection for which there is no evidence of its presence or incubation at the time of admission or any infection acquired within 72 hours of admission to Hospital $(3,4,5)$. World Health Organization published a guide to prevent HIV transmission in health 
facilities. All documents uniformly state that the basic principle of the UP (Universal Precaution) is to assume that all blood and body fluids are potentially infections, regardless of whether they originate from a patient or healthcare workers, their blood borne infection status and their known HIV antibody status. Therefore, precautions should be applied in the care of all patients (6). Study revealed that at any time over 1.4 million patients worldwide will have infectious complications acquired in the hospital. This study, most likely do not reflect the current situation because at that time the HIV/AIDS epidemic was just beginning. Moreover, the study did not include any countries of Africa where nosocomial infection rates are much higher (7). "Universal Precautions" are designed to prevent health care staff being exposed to blood and body fluids by applying the basicprinciple of infection control through hand washing, utilization of appropriate protective barriers, such asgloves, mask, gown and safe handling of needles(7).

Nosocomial or hospitalized acquired infections are infections appearing in a patient in a hospital or other health care facility in whom the infection was not present or incubating at the time of admission. Such infection manifest within 72 hoursror more fter admission (8). Over all national prevalence rates have been described as ranging between 3.5 and $9.9 \%$ and Hospital surfaces and nursing staff are the most prevalent sources of Nls (Nosocommial Infections) (9). Hospital associated infection which has a worldwide distribution remains a major cause of deaths among hospitalized patients (10). The spread of antibiotic resistance genes may becausally related to the overuse of antibiotics in human health care and in animal feeds, increased use of invasive devices and procedures, a greater number of susceptible hosts, and lapses in infection control practices leading to increased transmission of resistant organisms.

The resistance gene sequences are integrated by recombination into several classes of naturally occurring gene expression cassettes and disseminated within the microbial population by horizontal gene transfer mechanisms: transformation, conjugation or transduction (11). Infection in health care facilities is a major public health problem in most developing countries like Ethiopia. Currently the overall incidence of health care associated infection has been increased and burden of these infections is staggering. Thus, it is important to identify the level of health care workers knowledge, attitude and practice in study area and identifying gaps for better intervention(12). Despite the knowledge that contaminated hand splay an important role in the transmission of health care associated pathogens, and that hand hygiene $(\mathrm{HH})$ reduces the transmission of these organisms, health care worker's adherence with $\mathrm{HH}$ is poor (13). Most nosocomial infections are thought to be transmitted by the hands of health care workers and Implementation of multifaceted interventional behavioral hand hygiene program with continuous monitoring and erformancefeedback, increasing the supplies necessary for HW and institutional support are important for improving the compliance of hand hygiene guidelines (14). Proper glove utilization and appropriate hand washing practice has paramount importance to reduce and prevent hospital acquired infection. This can be achieved when health care worker have adequate knowledge and practice and compliance with hand washing and glove utilization. In Shenen Gibe hospital there has no formal research conducted on this subject matter, and therefore it is necessary to conduct abaseline study to determine the compliance of hand washing practice and gloe utilization with the standard, so that compliance with the standard was recommended. The purpose of this study was to investigated the knowledge and practice of hand washing and glove utilization among the health care workers of Shenen Gibe Hospital, South West Ethiopia.

\section{Methods and Materials}

\subsection{Study Area and Period}

The study was conducted from February 13,2014 in Shenen Gibe Hospital located in South West Ethiopia, Jimma Zone of Oromia Regional Stateat Jimma Town. The Hospital was established in 2012.The Hospital provides service to both in patient and out patients. It has 50 inpatient beds with different departments including Medical, Surgical, Pediatrics wards, Obstetrics and Gynecology. The general staff composition of the hospital was 47 Health care professionals, in which 1 Emergency Surgeons, 6 Medical Doctors. 24 Nursing staff and 16 other health personnel. The Nursing staff varies from B.Sc. holder to the clinical Nurse. There were 3 professional Nurse or B.Sc., 21 registered Clinical Nurse, 8 Midwifery Nurse. In addition to this there were 5 laboratory technician and 3 Anesthetists.

\subsection{Study Design}

The study design was adescriptive cross sectional study to assess the knowledge and practice of hand washing and gloveutilization among health care providers of Shenen Gibe hospital. The source populations for this study were health care providers of Shenen Gibe hospital. The study populations for this study were health care providers who are willing to participate in the study at Shenen Gibe Hospital. Voluntary, health care professionals who were available during data collection were included. Study population who were not available during data collection and involuntary participants were excluded from the present study.

\subsection{Study Population}

The source populations for this study were health care providers of Shenen Gibe Hospital. The study populations for this study were health care providers who are willing to participate in the study at Shenen Gibe Hospital.

\subsection{Sample Size}

The sample size was determined by using a single population proportional formula considering by the following 
assumptions: No enough staffs regarding this study sampling need. Therefore, Purposive sample taken.

\subsection{Sampling Techniques and Procedure}

As the number of the study population is not large enough to require sampling, all health care providers of Shenen Gibehospital were included in the study. Therefore, .Universal sampling method was used. As a result all the 47 health care providers of the Hospital had responded to the questionnaires.

\subsection{Instruments and Measurements}

Pre tested and structured self-administered questionnaire was used. Translation of instrument is made from English language to local Oromifa Language and back to English language by different experts who are familiar on the field of area and blind to the origina lversion of the questionnaire(English version) inorder to facilitateresponses to underline questions and keep the original meaning of the instrument. The instrument contains three parts: Socio demographic status of the responses (08 items), practice on hand washing and utilization of glove(12items), knowledge on hand washing and utilization of glove (11items), and general reasons health workers wore gloves and it's limitation (5items). The principal investigator conducted the data collection personally. A structured questionnaire developed was pre-tested ten days before launching the final data collection on $5 \%$ of the study population in Jimma University Specialized Teaching Hospital and required changes was made. There liability of the data collected was maintained to maximum possible. To ensure there liability and validity of data the principal investigator put the maximum effort. In this regard experts on the subject matter were contacted and the questionnairere viewed to obtain the desired variables. The data collected was kept in a lockablec abinet not accessible to anyone other than the principal investigator. In addition the respondents were not allowed to take home the questionnaires sothat noreferences made sothat their actual knowledge at the time of the study was analyzed (avoidbias). In addition, each participant was told and strictly followed by the principal investigator to respond to the questions by his/her own, without consulting anyone else, and that all the questions provided in the tool will be answered.

\subsection{Data Collection Procedure}

A structured questionnaire developed was pre-tested ten days before launching the final data collectionon $5 \%$ of the study population in Jimma University Specialized Teaching Hospital and required changes was made. There liability of the data collected was maintained to maximum possible. To ensure there liability and validity of data the principal investigator put the maximum effort. In this regard experts on the subject matter were contacted and the questionnairere viewed to obtain the desired variables. The data collected was kept in alockable cabinet not accessible to anyone other than the principal investigator. In addition the respondents were not allowed to take home the questionnaires so that no references made sothat their actual knowledge at the time of the study was analyzed (avoidbias). In addition, each participant was told and strictly followed by the principal investigator to respondtot he questions by his/her own, without consulting anyone else, and that all the question sprovided in the tool will be answered.

\subsection{Study Variable}

\subsubsection{Dependent Variable}

- Knowledge, Practice, Hand-Washing, Glove Utilization

\subsubsection{Independent Variable}

- Age, Sex, Socioeconomic status, religion place of living ,Ethnicity and Marital status

\subsection{Data Processing, Analysis and Presentation}

After the completion of the data collection process the required data was categorized and recorded. The data was compiled and the variables calculated and analyzed using manual hand scientific calculators. After analysis the results was presented in tables, figures or graphic forms.

\subsection{Operational Definition}

Knowledge is defined as having adequate understanding about the topic to which the person responds as evidenced by earning scores of $75 \%$ or more in the questions provided regarding knowledge.

- Knowledgeable: Earning Score of $75 \%$ and above on the Knowledge questions.

- Not knowledgeable: Earning score less than $75 \%$ on the knowledge questions.

- $\quad$ Practice of hand-washing and glove utilization .Practice is defined as anact of performing a given procedure (s ) according to a set standard.

- Good practice: Earning score of $75 \%$ and more on the practice questions.

- Poor practice: earnings core less than $75 \%$ on the practice questions.

\subsection{Data Quality Control}

To ensure the quality of data, formerly the instruments were pre tested. The pretest was conducted in $5 \%$ of the participants at randomly selected study area away from the study areas. Training was given for the data collectors and supervisors before the actual data collection. Every day after data collection, instruments were reviewed and checked for completeness, accuracy and clarity by the supervisors and principal investigator.

\subsection{Ethical Consideration}

Prior to the commencement of the study it was ethically cleared by The Research and Publication Office of Jimma University and Shenen Gibe Hospital Administration. In 
addition, the respondents were explained about the study and were request for their willingness to fill the questionnaires. The names of the respondents were not included in the questionnaire and in the results. All the filled tools were kept i n strict confidentiality in a lockable cabinet.

\section{Result}

This study was across-sectional study conducted among health care providers of Shenen Gibie Hospital on knowledge and practice of hand washing and glove utilization. The findings of the study were presented in this section.

\subsection{Demographics of the Study Population}

All the 47 staffs have responded to the questionnaire and the distribution of the staff was: $7(14.8 \%)$ physicians, $3(6.4 \%)$ were B.Sc nurses, 21(44.8\%) was clinical nurses, $8(17 \%)$ were midwifery nurse, $5(10.6 \%)$ lab tech. and the rest were anesthetist3(6.4\%).The gender distribution of the respondents showed that $15(31.9 \%)$ were males and 32(68.1\%) were females. This is shown in Table1 and Figure1.

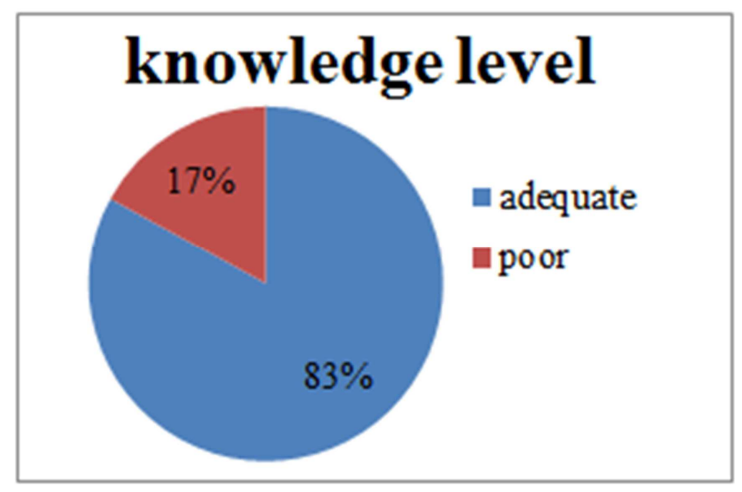

Figure 1. Distribution of knowledge status of Shenen Gibe Hospital staff on hand washing and glove utilization, 2014.

Table 1. Frequency distribution of study population by occupation and sex in Shenen Gibe Hospital ,2014.

\begin{tabular}{|c|c|c|c|c|c|c|}
\hline \multirow{2}{*}{ Category of staff } & \multicolumn{2}{|c|}{ Male } & \multicolumn{2}{|c|}{ Female } & \multicolumn{2}{|c|}{ Total } \\
\hline & No & $\%$ & No & $\%$ & No & $\%$ \\
\hline $\begin{array}{l}\text { Physicians(Emergency } \\
\text { Surgeon \& } \\
\text { medicaldoctor) }\end{array}$ & 5 & 10.6 & 2 & 4.25 & 7 & 14.85 \\
\hline Nurse(BSC) & 2 & 4.25 & 1 & 2.15 & 3 & 6.4 \\
\hline Clinical Nurse & 2 & 4.25 & 19 & 40.4 & 21 & 44.8 \\
\hline Midwifery nurse+ & 2 & 4.25 & 6 & 12.8 & 8 & 17 \\
\hline LabTech. & 3 & 6.4 & 2 & 4.25 & 5 & 10.65 \\
\hline Anesthetist & 1 & 2.15 & 2 & 4.25 & 3 & 6.4 \\
\hline Total & 15 & 31.92 & 32 & 68.1 & 47 & 100 \\
\hline
\end{tabular}

The service year of the respondents was calculated and $32(68.06 \%)$ served $0-5$ years, $11(23.42 \%)$ of the $\mathrm{m}$ have served 6-10 years, and 4(8.52\%) of them have served from 11-15 years. The statically analysis whether years of service affects the practice of hand washing and glove utilization revealedthere is no association between years of service and the practice of hand washing and glove utilization. It means according to this study the practice of hand washing and glove utilization is independent of the years of service.

Table 2. Socio demographic distributions of respondents in Shenen Gibe Hospital,2014.

\begin{tabular}{|c|c|c|c|c|c|c|c|}
\hline \multirow{2}{*}{ Category } & & \multicolumn{2}{|c|}{ Male } & \multicolumn{2}{|c|}{ Female } & \multicolumn{2}{|c|}{ Total } \\
\hline & & No & $\%$ & No & $\%$ & No & $\%$ \\
\hline \multirow{3}{*}{ Age } & $20-29$ & 10 & 21.27 & 23 & 48.94 & 33 & 70.21 \\
\hline & $30-39$ & 4 & 8.51 & 9 & 19.15 & 13 & 27.66 \\
\hline & $40-49$ & 1 & 2.13 & - & - & 1 & 2.13 \\
\hline \multirow{4}{*}{ Ethnicity } & Oromo & 14 & 29.78 & 17 & 36.17 & 31 & 65.95 \\
\hline & Amhara & 1 & 2.13 & 9 & 19.15 & 10 & 21.28 \\
\hline & Tigre & - & - & 1 & 2.13 & 1 & 2.13 \\
\hline & Others & - & - & 5 & 10.64 & 5 & 10.64 \\
\hline \multirow{4}{*}{ Religion } & Orthodox & 2 & 4.26 & 17 & 36.17 & 19 & 40.43 \\
\hline & Muslim & 6 & 12.76 & 8 & 17.07 & 14 & 29.83 \\
\hline & Protestant & 6 & 12.76 & 6 & 12.76 & 12 & 25.52 \\
\hline & Others & 1 & 2.13 & 1 & 2.13 & 2 & 4.24 \\
\hline \multirow{3}{*}{ Maritalstatus } & Single & 6 & 12.26 & 16 & 34.04 & 22 & 46.3 \\
\hline & Married & 8 & 17.03 & 14 & 29.78 & 22 & 46.3 \\
\hline & Divorced & 1 & 2.13 & 2 & 4.26 & 3 & 6.39 \\
\hline \multirow{3}{*}{ Yearofservice } & $0-5$ years & 10 & 21.27 & 22 & 46.79 & 32 & 68.06 \\
\hline & 6-10years & 3 & 6.39 & 8 & 17.03 & 11 & 23.42 \\
\hline & $\begin{array}{l}11- \\
15 \text { years }\end{array}$ & 2 & 4.26 & 2 & 4.26 & 4 & 8.52 \\
\hline
\end{tabular}

\subsection{Knowledge Versus Practice of Hand Washing and Glove Utilization}

The knowledge of the respondents was assessed and categorized as poor and adequate. Based on this the knowledge status of all staff is shown in Figure1.

\subsection{Knowledge Versus Practice of Hand Washing and Glove Utilization}

The knowledge of the respondents was assessed and categorized as poor and adequate. Based on this the knowledge status of all staff is shown in Figure2.

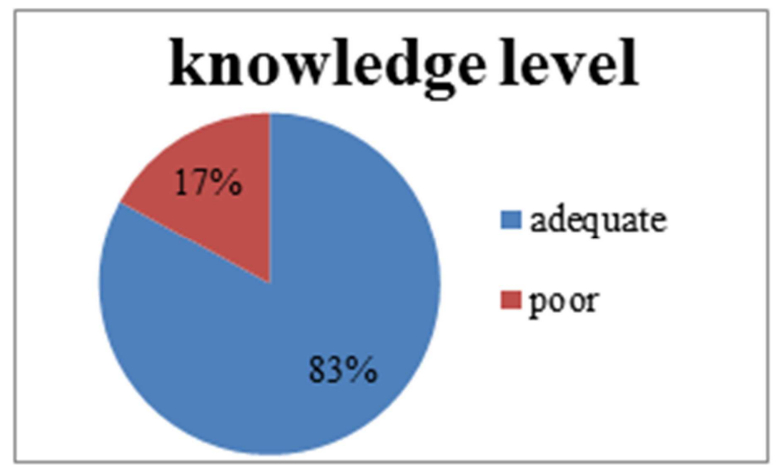

Figure 2. Distribution of knowledge status of Shenen Gibe Hospital staff on hand washing and glove utilization, 2014.

From 47 respondents, 32(68.08\%) of them have adequate practice of hand washing and glove utilization; and $15(31.92 \%)$ of them have inadequate practice of hand washing and gloveutilization. This reveals that the majority of the study subjects practice hand washing and glove utilization adequately. As summarized in Table 3,39(82.97\%) have adequate knowledge of hand washing and glove 
utilization and $8(17.03 \%)$ have inadequate knowledge of hand washing and glov eutilization. From all study subjects, $29(61.70 \%)$ have both adequate knowledge and adequate practice of hand washing and glove utilization; whereas $10(21.27 \%)$ have adequate knowledge but poor practice of hand washing and glove utilization, while $3(6.38 \%)$ have in adequate knowledge but have adequate practice of hand washing and glove utilization. The rest of the study subjects $5(10.63 \%)$ have inadequate knowledge and inadequate practice of hand washing and glove utilization. This study shows that the majority have adequate knowledge and adequate practices of hand washing and glove utilization.

Table 3. Frequency distribution of knowledge of hand washing and glove utilization vs. practices of the study subjects in Shenen Gibe hospital,2014.

\begin{tabular}{|c|c|c|c|c|c|c|}
\hline \multirow{3}{*}{ Knowledge of hand washing and glove utilization } & \multicolumn{4}{|c|}{ Practice of hand washing \& glove utilization } & \multirow{2}{*}{\multicolumn{2}{|c|}{ Total }} \\
\hline & \multicolumn{2}{|c|}{ Adequate } & \multicolumn{2}{|c|}{ Poor } & & \\
\hline & No & $\%$ & No & $\%$ & No & $\%$ \\
\hline Adequate & 29 & 61.70 & 10 & 21.27 & 39 & 82.97 \\
\hline Poor & 3 & 6.38 & 5 & 10.63 & 8 & 17.03 \\
\hline Total & 32 & 68.08 & 15 & 31.92 & 47 & 100 \\
\hline
\end{tabular}

\subsection{Hand Washing and Glove Utilization Practice Versus Previous Training}

This study showed that $17(36.17 \%)$ of the respondents had taken training related to hand washing and glove utilization, from these,14(29.79\%) adequately practice hand washing and glove utilization and the rest 3(6.38\%) had practiced hand washing and glove utilization inadequately. On the other hand,30(63.83\%) of the respondents did not take any training or pre-training to hand washing and glove utilization and from these $18(38.3 \%)$ were found to have adequate practice of hand washing and glove utilization while $12(25.53 \%)$ have in adequate hand washing and glove utilization. Table 4 summarizes this. Thestatistical analysis of these study showed that those who didn't taken pre-training had adequate knowledge and practice of hand washing and glove utilization than those who had taken pre-training on hand washing and glove utilization.

Table 4. Frequency distribution of hand washing and glove utilization practice and training of the participants on the subject in Shenen Gibe Hospital,2014.

\begin{tabular}{|c|c|c|c|c|c|c|c|c|c|c|}
\hline \multirow{3}{*}{ Respondents } & \multicolumn{4}{|c|}{$\begin{array}{l}\text { Adequate Hand washing \& Glove Utilization } \\
\text { Practice }\end{array}$} & \multicolumn{4}{|c|}{$\begin{array}{l}\text { Poor Hand Washing \& Glove Utilization } \\
\text { Practice }\end{array}$} & \multirow{2}{*}{\multicolumn{2}{|c|}{ Total }} \\
\hline & \multicolumn{2}{|c|}{ Training } & \multicolumn{2}{|c|}{ NoTraining } & \multicolumn{2}{|c|}{ Training } & \multicolumn{2}{|c|}{ NoTraining } & & \\
\hline & No & $\%$ & No & $\%$ & No & $\%$ & No & $\%$ & No & $\%$ \\
\hline Physician & 2 & 4.26 & 4 & 8.52 & - & - & 1 & 2.13 & 7 & 14.85 \\
\hline Professional Nurse & 1 & 2.13 & 1 & 2.13 & - & - & 1 & 2.13 & 3 & 6.4 \\
\hline Clinical Nurse & 8 & 17 & 7 & 14.85 & 2 & 4.26 & 4 & 8.52 & 21 & 44.8 \\
\hline Midwifery & 2 & 4.26 & 2 & 4.26 & 1 & 2.13 & 3 & 6.38 & 8 & 17 \\
\hline LabTech & 1 & 2.13 & 3 & 6.38 & - & - & 1 & 2.13 & 5 & 10.65 \\
\hline Anesthetist & - & - & 1 & 2.13 & - & -- & 2 & 4.26 & 3 & 6.39 \\
\hline Total & 14 & 29.79 & 18 & 38.3 & 3 & 6.39 & 12 & 25.53 & 47 & 100 \\
\hline
\end{tabular}

\subsection{Compliance with the Recommended Practices of Hand Washing and Glove Utilization}

From the total of 47 respondents, $24(51 \%)$ of the $\mathrm{m}$ have complied with the recommended procedures of hand washing and glove utilization, while $23(49 \%)$ of the m did not comply with the recommended procedures of hand washing and glove utilization; these study showed that most of the study subjects did comply with the recommended procedures of hand washing and glove utilization.

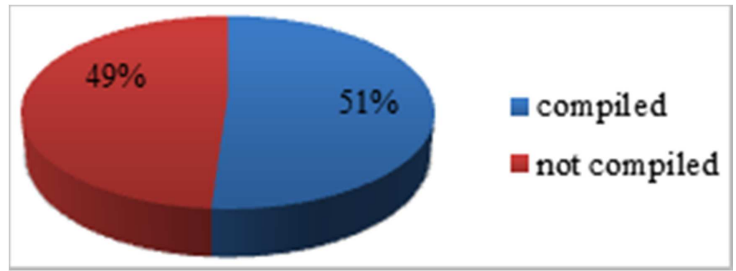

Figure 3. Compliance of Shenen Gibe Hospital health care providers with therecommended practices of hand washing and glove utilization, 2014.

\subsection{Educational Status and Compliance to the Recommended Procedures of Hand Washing and Glove Utilization}

Table 5. Compliance on the recommended practices of hand washing \& gloveutilization among staff at Shenen Gibe Hospital, 2014.

\begin{tabular}{lllllll}
\hline \multirow{2}{*}{ StudySubject } & \multicolumn{2}{l}{ Compiled } & \multicolumn{2}{l}{ NotComplied } & Total \\
\cline { 2 - 7 } & No & $\%$ & No & $\%$ & No & \% \\
\hline Physician & 4 & 57.1 & 3 & 42.9 & 7 & 100 \\
Nurse(BSc) & 2 & 66.6 & 1 & 33.4 & 3 & 100 \\
Clinical Nurse & 9 & 42.9 & 12 & 57.1 & 21 & 100 \\
Midwifery & 5 & 62.5 & 3 & 37.5 & 8 & 100 \\
LabTech & 3 & 60 & 2 & 40 & 5 & 100 \\
Anesthetist & 1 & 33.4 & 2 & 66.6 & 3 & 100 \\
Total & 24 & 51 & 23 & 49 & 47 & 100 \\
\hline
\end{tabular}

Investigation of how educational status affects compliance with the recommenced procedures of hand washing and glove utilization was done by comparing nurses with physicians with respect to their compliance with the recommended procedures. From 21, clinical nurses, 9(42.9\%) 
of them had complied and $12(57.1 \%)$ had not complied with the recommended procedures of hand washing and glove utilization. From 7, physician, 4(57.1\%) of them complied and $3(42.9 \%)$ was not complied. The details of compliance with recommended guideline for all staff is shown in Table 5.

\subsubsection{Antiseptic Use}

Out of the 47 respondents, 31(65.95\%) uses soap for hand washing, and 26(55.32\%) uses alcohol, savlon and more thanone antiseptic agents where more than one answer was common.. From this finding soap is more used than most frequentlyused antiseptic agent. Some respondents showed the unavailability of soap sometimes and only wash with water alone.

\subsubsection{Hand Drying Method}

The study revealed that the study population use Common towel, air, gauze and out of them 23(48.9\%) use air,19(40.4\%) use common towel11(23.4)gauze user where more than one answer was common. After washing the hand proper hand drying is the recommended procedure and the use of improper drying can cause the transfer of pathogens from one person to another through the hands of health providers.

\section{Discussion}

As detailed in the results about the knowledge of staff on glove utilization and hand hygiene was assessed and $82.9 \%$ of all staffs have adequate knowledge while the rest $17.03 \%$ have inadequate knowledge. This result was comparable but slightly higher than the study finding done at Jimma University hospital nursing staff on hand washing practice in which case $71.6 \%$ of the staff had adequate knowledge while the rest 28.4 did not have(7). This finding shows that shenen gibe hospital staff knowledge on glove utilization and hand hygiene was good though attentionis required to improve the knowledge of those staff that has poor knowledge. Concerning Shenen Gibe Hospital staff on hand hygiene and glove utilization their practice is good. As the result shown about $68.08 \%$ of the staff properly practices good hand hygiene and glove utilization while the rest $31.92 \%$ were not. This result is similar to the study finding in that of a tertiary care pediatric hospital in Canada, Toronto, Jimma university nursing staff and in University of Pittsburgh Medical center which showed a hand hygiene practice rate of $61 \%, 43 \%$ and $57 \%$ respectively $(7,15)$, but higher than Turkey where only $26.9 \%$ of the health care worker sshowed compliance to hand washing both before and after the use of glove in specific procedure(15). This result was higher than hand washing compliance of health care workers before glove utilization in medical and cardiac surgery ICU in Virginia hospital which showed $3 \%$ and $13 \%$ respectively in each department (6). The possible reasons for such a high compliance to hand washing and glove utilization practice is that, the staff have received recent training on such topic and have adequate knowledge regarding hand washing and glove utilization. In addition lack of standard operating procedures and lack of strict rules to implement such by the department leaders/hospital management could contribute to suchlow compliance health professional. When educational status and compliance was compared, compliance to the set procedures was indirectly related to educational status. The compliance of nurses was better than physcians and other health professionals. This result was similar to the study finding in tertiary care hospital in Canada where the compliance rate of nurses in the hospital was $63 \%$ which was higher than physicians in the same hospital which accounts $44 \%$. This may be because of hand washing and glove utilization was given for nurses as a course and nurses have cloth contact with the patient as compared physicians (15).

\section{Strength and Weakness of the Study}

The study is interesting in which authors' explored knowledge and practices of hand washing and glove utilization among health care providers, less explored in previous studies. However, the relatively small sample size and the single center study limit the generalizability of the study findings.

\subsection{Strength of the Study}

- The data was collected using a structured selfadministered pre-tested questionnaire

- Adequate sample size was applied according to single population proportion formula

- Data collators were health professional

\subsection{Weakness of the Study}

- During this study there may be observational bias

- There were financial constraint while conducting the Study

- All respondents were interviewed but some of them observed during the study period

- Some of the respondents were volunteer during data collection

\section{Conclusion}

Based on the study results the following can concluded: Majority of the study subjects have adequate knowledge about hand washing and gloveutilization. Most of the study subjects have a dequate practice of about hand washing and glove utilization. Knowledge of hand washingand glove utilization has direct relation to training and practice of hand washing and glove utilization. Most of the respondents were compiled with there commended procedures of hand washing and glove utilization. Educational status was indirectly related to recommended procedure of hand washing and glove utilization. Regular practice of hand washing and glove utilization requires regular supplieslike soap, water, dry\& clean to wel and acceptable glove depending on the type of procedure to perform at all time. Therefore Oromia Health Bureau should facilitate these facilities in the hospital wards. 


\section{Author's Contributions}

$B S$, have made substantial contributions to beginning and design, collection of data, analysis and interpretation of data and in drafting the manuscripts and correcting the comment given by the advisors.

$A D$ involved in revising the research paper and the manuscript critically for important intellectual context and approval of the final version to be published and participated in its design and coordination. Heparticipated in the approval and funding process, participated in the design of the study participated in its design and coordination.

$J J$, involved in revising the research paper and the manuscript critically for important intellectual context and approval of the final version to be published and participated in its design and coordination.

$A A G$ had greater contribution in reviewing the manuscript English and topography. And helped to draft the manuscript.

$Y Y A$ had greater contribution in reviewing the manuscript English and topography. And helped todraft the manuscript.

$B A$ had greater contribution in reviewing the manuscript English and topography. And helped to draft the manuscript.

\section{Acknowledgements}

We are thankful to all our department technical staffs for their excellent technical support. We are grateful to all the participants for their cooperation and Jimma University for financial support.

\section{References}

[1] Haileamlak, A (2011). Preventing healthcare associated infections. Ethiop J Health Sci. 21(1): 7-8.

[2] David OM, Famurewa O. Toward effective management of Nosocomial infections in Nigerian Hospitals-A review. Academic Arena, 2010;2(5):1-7.

[3] Takahashi, I., Osaki, Y., Okamoto, M., Tahara, A., Kishimoto,, T(2009). The current status of hand washing and glove use among care staff in Japan: its association with the education, knowledge, and attitudes of staff, and infection control by facilities. Environ Health Prev Med. 2009 Nov; 14(6): 336-344. doi: 10. 1007/s12199-009-0103-z

[4] Watson, C., Annus, A. (2013) Intravenous diuretic delivery in the home Nursing Times, 109(14):1-5.
[5] Sile, A(2005). Issues and Innovations in Nursing Practice, Journal of Advanced Nursing, Blackwell Publishing Ltd, 51(3), 208-216.

[6] Bachok, N., Nordin, RB., Awang, CW., Ibrahim, NA., Naing, L (2006). Prevalence and associated factors of head lice infestation among primary schoolchildren in Kelantan, Malaysia. Southeast Asian J Trop Med Public Health. 2006; 37(3):536-43.

[7] Omolara Gbonjubola Uti, Gbemisola A. Agbelusi, Sonny Olukayode Jeboda, Eyitope Ogunbodede , 2009, Infection Control knowledge and Practices related to HIV among Nigerian Dentists, J infect Dev Ctries, 3 (8): 604-610

[8] David OM, Famurewa O. Toward effective management of Nosocomial infections in Nigerian Hospitals-A review. Academic Arena, 2010;2(5):1-7.

[9] Jalalpoor S, Kasra, Kerman Shahi, NoohiA, Zarkesh H. $\beta$ lactamase and $\mathrm{S}$ layerproduction in some of isolated pathogen bacteria from clinical and environmental Hospital samples MSC thesis, Iran, Tehran, Islamic Azad University, Science and Research Branch, Tehran. 2007; 105-157.

[10] Olajubu FO, Osinupebi OA, LawalI, Obadina B, DejiAgboola AM. Pattern of hospital associated infections in a teaching hospital in Nigeria. Asian Pacific Journal of Tropical Disease. 2012:S869-S873.

[11] Hunter PR, Wilkinson DC, Catling L A, Barker GC. MetalAnalysis of experimental data concerning antimicrobial resistance gene transfer rates during conjugation. Applied and Environmental Microbiology. 2008; 74(19):6085-6090.

[12] Gulilat, K., Tiruneh, G(2014). Assessment of knowledge, attitude and practice of health care workers on infection prevention in health institution Bahir Dar city administration. Science Journal of Public Health2014; 2(5): 384-393Published online August 10, 2014 (http://www. sciencepublishinggroup. $\mathrm{com} / \mathrm{j} / \mathrm{sjph}$ ) doi: 10. 11648/j. sjph. 20140205. 13

[13] Dixit, D., Hagtvedt, R., Reay, T., Ballermann, M., Forgie, S(2012). Attitudes and beliefs about hand hygiene among paediatric residents: a qualitative study. BMJ Open 2; 2:e002188 doi: 10. 1136/bmjopen-2012-002188.

[14] Elaziz, K(2009). Assessment of knowledge, attitude and practice of hand washing among health care workers in Ain Shams University hospitals in Cairo. J prev med hyg; 50: 1925

[15] Sacar, S., Turgut. H., Kaleli, I., Cevahir, N., Asan, A., M. Sacar, M., Tekin, K(2006). Poor Hospital Infection control practice in hand hygiene, glove utilization, and usage of tourniquets. Antimicrobial Journal of Infection Control, 34: 606-609. 